\title{
Outcomes of Infected Cardiovascular Implantable Devices in Dialysis Patients
}

\author{
Oluwaseun Opelami ${ }^{\mathrm{a}}$ Ankit Sakhuja ${ }^{\mathrm{b}}$ Xiaobo Liu ${ }^{\mathrm{c}}$ W.H. Wilson Tang ${ }^{\mathrm{d}, \mathrm{e}}$ \\ Jesse D. Schold ${ }^{c, e}$ Sankar D. Navaneethan ${ }^{\text {a, e }}$ \\ ${ }^{a}$ Department of Nephrology and Hypertension, Glickman Urological and Kidney Institute, Cleveland Clinic, \\ Cleveland, Ohio, b ${ }^{b}$ Department of Nephrology and Hypertension, University of Michigan, Ann Arbor, Mich., ' Department \\ of Quantitative Health Sciences, Cleveland Clinic, ${ }^{d}$ Department of Cardiovascular Medicine, Heart and Vascular Institute, \\ Cleveland Clinic, and ${ }^{\mathrm{e}}$ Cleveland Clinic Lerner College of Medicine of CWRU, Cleveland Clinic, Cleveland, Ohio, USA
}

\section{Key Words}

Dialysis $\cdot$ Infection $\cdot$ Mortality $\cdot$ Hospitalization

\begin{abstract}
Background/Aims: Dialysis patients are at a higher risk for cardiovascular implantable electronic device (CIED) infection-related hospitalizations. We compared the outcomes and cost for dialysis and non-dialysis patients hospitalized with CIED infections. Methods: We conducted a retrospective analysis of the Nationwide Inpatient Sample (NIS) discharge records from 2005 to 2010. Patients with CIED infections were identified using ICD-9 codes for device-related infections or device procedure along with bacteremia, endocarditis or systemic infection. Dialysis patients were identified using ICD-9 codes. Multivariable logistic and linear regressions were performed to examine in-hospital mortality, length of stay and cost. Results: Of the 87,798 estimated hospitalizations with CIED infections, 6,665 (7.6\%) were dialysis patients. CIED-infection-related hospitalization has increased over time among dialysis patients. In-hospital mortality was higher among dialysis patients $(13.6 \%$ vs. $5.9 \%, p<$ 0.001 ). In the multivariable model, dialysis patients had higher odds of in-hospital mortality (odds ratio $1.98 ; 95 \% \mathrm{Cl}: 1.6$, 2.4) compared to the non-dialysis group. Dialysis patients had a longer median length of stay ( 12 days vs. 7 days, $p<$ 0.001 ) and majority required extended care facility upon dis-
\end{abstract}

charge $(51.2 \%$ vs. $35.0 \%, \mathrm{p}<0.001)$ compared to the nondialysis group. Dialysis status was associated with $50.3 \%$ increased cost of hospitalization ( $p<0.001)$. Conclusion: CIEDinfection related hospitalization is increasing among patients undergoing dialysis and is associated with higher in-hospital mortality, longer hospital stay and higher costs of hospitalization. Future studies should examine the reasons for such a high risk and find means to improve outcomes in dialysis population.

(c) 2014 S. Karger AG, Basel

\section{Background}

Dialysis patients are known to have a higher burden of cardiovascular disease related to coronary atherosclerosis, left ventricular hypertrophy, myocardial fibrosis, volume overload and arrhythmias [1-4]. Overall, cardiovascular disease remains the leading cause of death in this population and specifically, $27 \%$ of all deaths among prevalent dialysis patients are attributed to arrhythmiarelated sudden cardiac death (SCD) [5]. Although most major randomized controlled trials evaluating the benefits of cardiovascular implantable electronic devices (CIEDs) such as implantable cardiac defibrillators (ICD) and cardiac resynchronization therapy (CRT) devices excluded ESRD patients, ICD implantation following an

\section{KARGER}

E-Mail karger@karger.com

www.karger.com/ajn
(C) 2014 S. Karger AG, Basel

0250-8095/14/0403-0280\$39.50/0
Sankar D. Navaneethan, MD, MPH

Department of Nephrology and Hypertension

Glickman Urological and Kidney Institute, 9500 Euclid Avenue - Q7

Cleveland Clinic, Cleveland, Ohio 44195 (USA)

E-Mail navanes@ccf.org 
SCD event has been shown to be independently associated with a $42 \%$ reduction in risk of death in this population $[6,7]$. A recent meta-analysis also reported mortality benefit in chronic kidney disease (CKD) patients following CRT [7].

Even though not proven, dialysis patients may therefore represent a unique group of patients for which CIEDs may potentially provide substantial benefits. However, a few studies have demonstrated an increasing trend of CIED infections in the general population, which has been out of proportion to the rate of device implantations and these infections have been associated with higher in-hospital mortality $[8,9]$. The expanded indications for CIEDs, changing patient demographics and the placement of these devices in high-risk patients with greater comorbidity burden may be contributing to this trend [8]. Dialysis patients are at a high risk of complications following implantation of CIEDs, including device lead dislodgment, dialysis access thrombosis, bleeding and infections [1014]. Due to multifactorial reasons, they are more prone to develop endovascular infections [15] and several smaller studies have reported an increased risk of CIED-related infections in this population. Therefore, we sought to describe the characteristics, incidence, outcomes and costs for dialysis patients hospitalized with CIED-infections using a large national inpatient database.

\section{Methods}

\section{Study Design and Data Source}

This retrospective analysis was conducted using the Healthcare Cost and Utilization Project-Nationwide Inpatient Survey (NIS), an administrative database created by the Agency for Healthcare Research and Quality (AHRQ) and represents the largest all-payer inpatient care database publicly available in the United States. The database represents data from the $20 \%$-stratified sample of US community hospitals and to facilitate the projection of national estimates, both hospital and discharge weights are provided, along with the information necessary to calculate the variance of estimates. Demographic variables (e.g., age, race, sex), payer characteristics and hospital characteristics such as teaching status, location (rural vs. urban), hospital size and region are available in the NIS database. Hospitals are considered teaching status if they have an American Medical Association-approved residency program, are a member of the Council of Teaching Hospitals, or have a fulltime or equivalent interns and residents to patient ratio of 0.25 or higher. Hospitals with a core-based statistical area type of metropolitan were categorized as urban and hospitals with a core-based statistical area type of rural were categorized as rural. The bed-size cutoff values are chosen so that approximately one-third of the hospitals in a given region, location, and teaching status combination would fall within each bed-size category (small, medium and large).

CIED Infections in Dialysis
Study Population

We queried the NIS database from 2005 to 2010, comparing dialysis vs. non-dialysis patients admitted with CIED infections. We identified patients with CIED infections using the International Classification of Diseases-9th Revision-Clinical Modification (ICD9-CM). Specifically, we used ICD-9-CM codes for device-related infection (996.61) or procedure code for device explanation (37.77, $37.79,37.89,37.99)$ along with evidence of infection such as sepsis (038, 785.59), bacteremia (790.7), bacterial endocarditis (421.0, $421.9,424.9)$, abscess/cellulitis (682.9) or fever (780.6) as had been used in previous literature [9]. ESRD patients were identified using the ICD-9-CM code for ESRD (585.6) (fig. 1). Dialysis patients were selected by excluding renal transplant patients (V42.0).

\section{Study Variables}

Age, sex, race and primary payer status were identified as factors using appropriate variables from the NIS database. The age was divided into five subgroups - 18-34, 35-49, 50-64, 65-79 and over 80 years. Hospital characteristics - teaching status, location, bedsize and region were also identified using appropriate NIS variables. We used the Deyo's modification of Charlson's comorbidity index to identify the burden of comorbidity [16]. Zip code details (as a proxy for socioeconomic status) are available in NIS and were categorized into quartiles with an additional level for missing values.

\section{Outcomes}

Outcome measures include incidence rates, all-cause in-hospital mortality, length of hospital stay, discharge disposition and costs associated with CIED-infection related hospitalizations. Hospital cost estimates were converted from hospital charges to costs by the 'cost-to-charge' ratio, provided by the Healthcare Cost and Utilization Project (HCUP). The HCUP cost-to-charge ratio is hospital and year specific.

\section{Statistical Analysis}

We used weights provided by the NIS to generate national estimates of the number of hospitalizations. The number of CIEDinfection-related hospitalizations per 100,000 populations was calculated by using the annual population estimates from the United States Census Bureau: American FactFinder (http://factfinder2. census.gov/faces/nav/jsf/pages/index.xhtml) and United States Renal Data System RenDER (http://www.usrds.org/render/xrender_home.asp). Direct standardization of age was performed to standard 2000 US standard population to estimate age-adjusted incidence rates. Similarly, we calculated age-adjusted incidence rates for CIED-infection-related hospitalizations for dialysis and non-dialysis groups from 2006 to 2010 . Since the ESRD code was introduced in 2005, the rates were very low in 2005 and thus we excluded the data from year 2005 for incidence rate calculation. Significance of trends for age-adjusted incidence over the years was assessed using linear regression.

The Chi-square test was used to evaluate the relationship between the categorical variables. Because the length of stay of survivors was not normally distributed, the $t$ test was performed on logtransformed values and median and inter-quartile ranges have been reported. We examined the risk factors for mortality in those admitted with CIED-related infections using weighted logistic regression in the multivariable model, which was adjusted for age, sex, race, primary payer, hospital teaching status, hospital location, hospital region, hospital volume (small, medium and large), hospital bed 


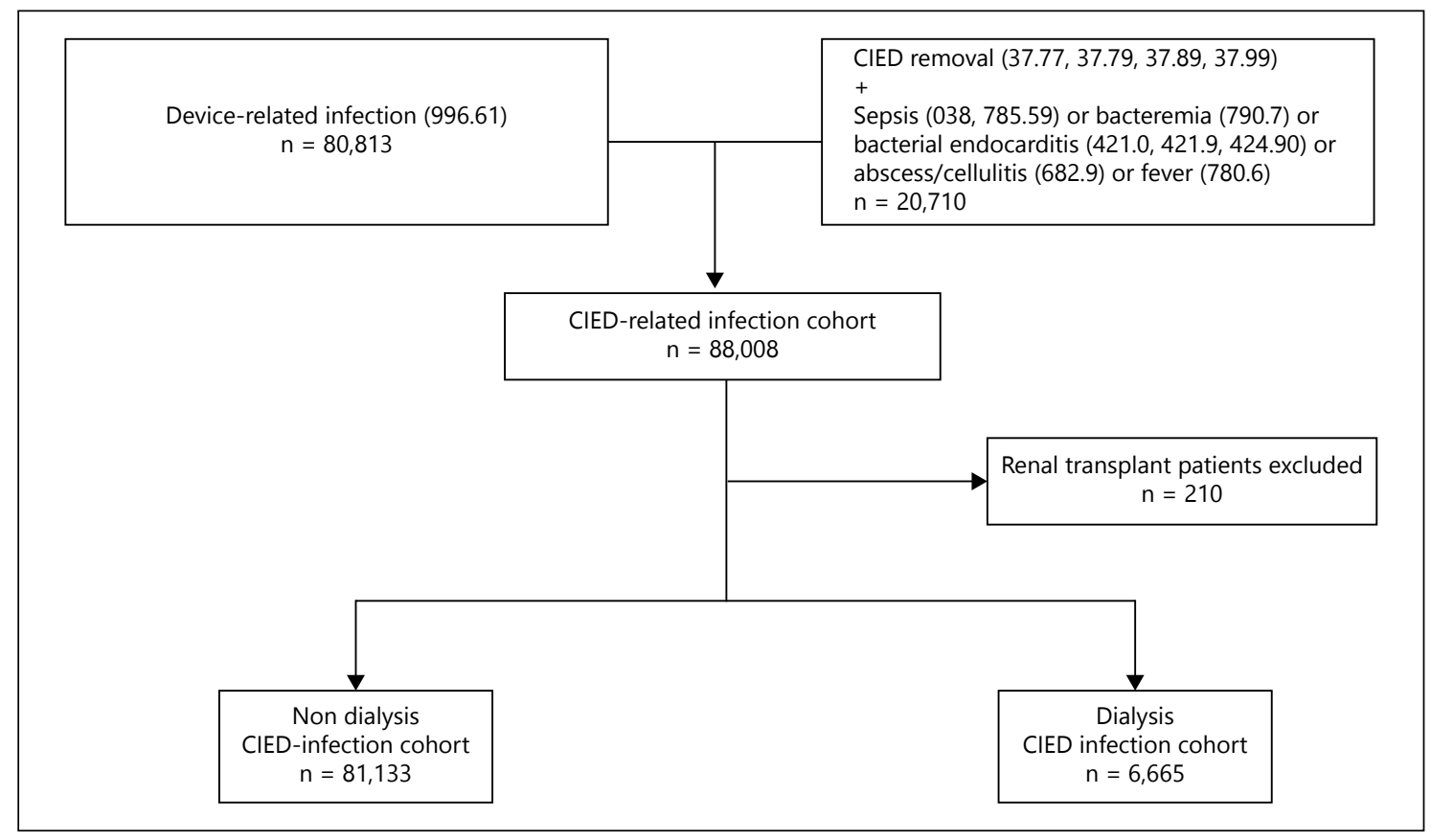

Fig. 1. Selection of study cohort.

size, zip code and the Charlson's comorbidity index score. These variables have been chosen because they were either reported to be associated with mortality or found to be clinically relevant. Similarly, for factors associated with the length of stay of survivors, a weighted multivariable regression analysis (adjusting for the same variables included in the mortality model) was performed. For this analysis, the length of stay was considered in log scale due to the skewed distribution. We also performed a weighted multivariable analysis using costs as log transformed variable to assess the differences between dialysis and non-dialysis patients. To control for variability by year, we used year as a predictor in all regression models. Disposition details were summarized for dialysis and non-dialysis groups. As information regarding race was missing in over $20 \%$ of discharges, we performed a sensitivity analysis for predictors of mortality by excluding missing values for race from analysis, a method that has been used in literature to overcome the issue of missing information regarding race from NIS database. We also reported the reimplantation rates (only during the same hospitalization) based on the ICD-9 codes for the cardiovascular devices. In addition, we also conducted subgroup analysis for various types of infections (i.e., sepsis, infective endocarditis, etc.) to assess if there were differences based on these diagnosis codes. All analyses were performed using the SAS 9.3 software (SAS Institute, Cary, N.C., USA). This study was approved by the Cleveland Clinic Institutional Review Board.

\section{Results}

\section{Patient Characteristics}

During the study period (2005-2010), there were 87,798 estimated hospitalizations for CIED-related infections, of which an estimated 6,665 (7.6\%) were dialysis patients. Dialysis patients were younger with $48.4 \%$ aged $<65$, compared with $39.7 \%$ of non-dialysis patients $(\mathrm{p}<0.001)$. There were higher proportions of African Americans (26.5\% vs. $8.3 \%)$ and Hispanics (8.0\% vs. $5.6 \%)$ among dialysis patients $(\mathrm{p}<0.001)$. Medicare was the primary payer for $80.3 \%$ of dialysis patients compared to $64.7 \%$ of non-dialysis patients $(\mathrm{p}<0.001)$. Other baseline patient characteristics (gender and other comorbid conditions), payer characteristics and hospital characteristics are presented in table 1.

\section{Incidence}

The overall unadjusted incidence of CIED-infectionrelated hospitalizations in the dialysis group and non-dialysis groups were 300.3 and 6.1 per 100,000 persons, respectively. As shown in figure 2, age-adjusted incidence rates of CIED-related infection were also higher in the dialysis population and the age-adjusted incidence of hospitalizations increased during the 5-year period (20062010) in the dialysis group (fig. 2).

\section{Mortality}

During the study period (2005-2010), unadjusted allcause in-hospital mortality was $13.7 \%$ for dialysis patients admitted with CIED-related infections, compared to $5.9 \%$ in the non-dialysis group ( $\mathrm{p}<0.001$; table 2$)$. In the multivariable model, dialysis patients had two times 
Table 1. Demographic characteristics of dialysis and non-dialysis patients admitted with cardiac device-related infections

\begin{tabular}{|c|c|c|c|}
\hline Variable & $\begin{array}{l}\text { Dialysis, } \mathrm{n}(\%)^{*} \\
(\mathrm{n}=6,665)\end{array}$ & $\begin{array}{l}\text { Non-dialysis, } \mathrm{n}(\%)^{*} \\
(\mathrm{n}=81,133)\end{array}$ & $\mathrm{p}$ value \\
\hline Age group & & & $<0.001$ \\
\hline $18-34$ & $229(3.4 \%)$ & $2,898(3.6 \%)$ & \\
\hline $35-49$ & $808(12.1 \%)$ & $8,465(10.4 \%)$ & \\
\hline $50-64$ & $2,191(32.9 \%)$ & $20,811(25.7 \%)$ & \\
\hline $65-79$ & $2,726(40.9 \%)$ & $29,715(36.6 \%)$ & \\
\hline$\geq 80$ & $711(10.7 \%)$ & $19,244(23.7 \%)$ & \\
\hline \multicolumn{4}{|l|}{ Gender } \\
\hline Female & $2,403(36.0 \%)$ & $25,571(31.5 \%)$ & $<0.001$ \\
\hline Race & & & $<0.001$ \\
\hline White & $2,727(40.9 \%)$ & $51,371(63.3 \%)$ & \\
\hline Black & $1,767(26.5 \%)$ & $6,709(8.3 \%)$ & \\
\hline Hispanic & $531(8.0 \%)$ & $4,509(5.6 \%)$ & \\
\hline Asian/Pacific Islander & $140(2.1 \%)$ & $1,056(1.3 \%)$ & \\
\hline Native American & $34(0.5 \%)$ & $441(0.5 \%)$ & \\
\hline Others & $144(2.2 \%)$ & $1,692(2.1 \%)$ & \\
\hline Missing & $1,321(19.8 \%)$ & $15,355(18.9 \%)$ & \\
\hline Primary payer & & & $<0.001$ \\
\hline Medicare & $5,353(80.3 \%)$ & $52,568(64.7 \%)$ & \\
\hline Medicaid & $479(7.2 \%)$ & $6,777(8.4 \%)$ & \\
\hline Private & $731(11.0 \%)$ & $18,037(22.2 \%)$ & \\
\hline Self-pay & $45(0.7 \%)$ & $1,593(2.0 \%)$ & \\
\hline No charge/others/missing & $57(0.8 \%)$ & $2,158(2.7 \%)$ & \\
\hline Hospital characteristics & & & 0.02 \\
\hline Teaching status & $4,284(64.3 \%)$ & $49,422(60.9 \%)$ & \\
\hline Location & & & 0.006 \\
\hline Urban & $6,381(96.2 \%)$ & $76,183(94.4 \%)$ & \\
\hline Bed Size & & & 0.004 \\
\hline Small & $614(9.1 \%)$ & $6,925(8.5 \%)$ & \\
\hline Medium & $974(14.5 \%)$ & $14,543(18.0 \%)$ & \\
\hline Large & $5,136(76.4 \%)$ & $59,665(73.5 \%)$ & \\
\hline Region of Hospital & & & 0.011 \\
\hline North east & $1,114(16.7 \%)$ & $14,463(17.8 \%)$ & \\
\hline Midwest & $1,692(25.4 \%)$ & $18,417(22.7 \%)$ & \\
\hline South & $2,443(36.7 \%)$ & $28,967(35.7 \%)$ & \\
\hline West & $1,002(15.0 \%)$ & $14,792(18.2 \%)$ & \\
\hline Missing & $79(6.2 \%)$ & $4,494(5.5 \%)$ & \\
\hline Charlson's score & $3.84 \pm 0.04$ & $1.86 \pm 0.01$ & $<0.001$ \\
\hline
\end{tabular}

* Estimates from weighted survey data, Rao-Scott chi-square test used for comparison.

Table 2. Comparison of outcomes of dialysis and non-dialysis patients admitted with cardiac device-related infections (unadjusted)

\begin{tabular}{lccc}
\hline Outcomes & $\begin{array}{l}\text { Dialysis, } \mathrm{n}(\%) \\
\left(\mathrm{n}^{*}=6,665\right)\end{array}$ & $\begin{array}{l}\text { Non-dialysis, } \mathrm{n}(\%) \\
\left(\mathrm{n}^{*}=81,004\right)\end{array}$ & $\mathrm{p}$ value \\
\hline All cause in-hospital mortality, \%* & $905(13.6 \%)$ & $4,783(5.9 \%)$ & $<0.001$ \\
LOS of survivors, days $^{\dagger}$ & $12(6,23)$ & $7(4,14)$ & $<0.001$ \\
Cost (dollars) $^{\ddagger}$ & $54,930(1,917)$ & $34,990(380)$ & $<0.001$
\end{tabular}

* Estimates from weighted survey data, Rao-Scott chi-square test used for comparison. ${ }^{\dagger}$ Median (IQR) from non-survey weighted data, $t$ test on log-transformed values using survey weighted data used for comparison. ‡ Mean (Standard error) from weighted survey data, $t$ test used for comparison. 


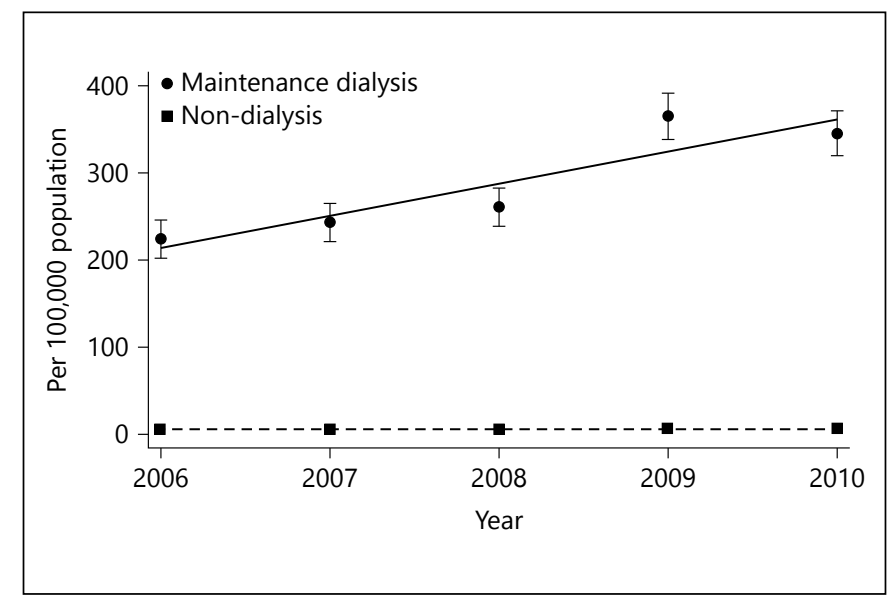

Fig. 2. CIED-infection-related hospitalizations over time among dialysis and non-dialysis patients.

higher odds of death, compared to non-dialysis patients (table 3). Other factors that were predictive of mortality were: age $>65$ years, higher comorbid disease burden and patients admitted to teaching hospitals in the recent years.

\section{Length of Stay and Disposition}

Among survivors, the unadjusted length of stay for dialysis patients was shorter ( $\mathrm{p}<0.001$; table 2 ). In the multivariable model, the dialysis status was associated with a $24 \%$ increased length of hospital stay when compared with non-dialysis patients hospitalized for CIED-related infections ( $\mathrm{p}<0.0001$ ). Among survivors, $51.2 \%$ of dialysis patients were transferred to skilled nursing facilities or other rehabilitation centers at the time of discharge, compared to $35.0 \%$ in the non-dialysis group (online suppl. table 1; for all online suppl. material, see www.karger. com/doi/10.1159/000366453).

\section{Cost}

After converting hospital charges to cost using the cost-to-charge ratio provided by the HCUP, dialysis patients had higher unadjusted costs associated with these hospitalizations than non-dialysis patients (table 2). In the multivariable model (with costs considered in log scale), dialysis patients had $31 \%$ higher cost of hospitalization than non-dialysis population $(\mathrm{p}<0.001)$.

\section{Reimplantation Rates}

Reimplantation rates for pacemaker and implantable cardioverter defibrillator were $2.1 \%$ among dialysis and $4.3 \%$ among non-dialysis population during the same hospitalization period.
Table 3. Predictors of mortality in patients hospitalized with CIEDrelated infections

\begin{tabular}{|c|c|c|c|}
\hline Factor & Odds ratio & $95 \% \mathrm{CI}$ & $\mathrm{p}$ value \\
\hline Dialysis vs. non-dialysis & 1.98 & $1.63-2.40$ & $<0.0001$ \\
\hline Age & & & 0.03 \\
\hline $35-49$ vs. $18-34$ & 1.18 & $0.77-1.83$ & \\
\hline $50-64$ vs. $18-34$ & 1.40 & $0.94-2.09$ & \\
\hline $65-79$ vs. $18-34$ & 1.55 & $1.03-2.34$ & \\
\hline$\geq 80$ vs. $18-34$ & 1.71 & $1.12-2.61$ & \\
\hline Gender & & & 0.08 \\
\hline Male vs. female & 0.89 & $0.78-1.02$ & \\
\hline Race & & & 0.84 \\
\hline Asian/Pacific Islander vs. White & 0.88 & $0.52-1.50$ & \\
\hline Black vs. White & 0.99 & $0.80-1.22$ & \\
\hline Hispanic vs. White & 0.90 & $0.69-1.19$ & \\
\hline Missing vs. White & 1.03 & $0.87-1.23$ & \\
\hline Native American vs. White & 0.87 & $0.35-2.18$ & \\
\hline Other vs. White & 1.29 & $0.88-1.89$ & \\
\hline Primary payer characteristics & & & 0.21 \\
\hline Medicaid vs. medicare & 1.31 & $1.02-1.68$ & \\
\hline No charge vs. medicare & 1.99 & $0.71-5.60$ & \\
\hline Other/missing vs. medicare & 1.34 & $0.89-2.02$ & \\
\hline Private insurance vs. medicare & 1.12 & $0.94-1.35$ & \\
\hline Self-pay vs. medicare & 1.27 & $0.78-2.07$ & \\
\hline Zip code (quartiles) & & & 0.72 \\
\hline First vs. fourth (highest) & 0.99 & $0.82-1.19$ & \\
\hline Second vs. fourth (highest) & 1.04 & $0.86-1.25$ & \\
\hline Third vs. fourth (highest) & 1.05 & $0.87-1.25$ & \\
\hline Missing vs. fourth (highest) & 0.77 & $0.49-1.22$ & \\
\hline Hospital characteristics & & & $<0.001$ \\
\hline Teaching vs. non-teaching & 1.30 & $1.13-1.49$ & \\
\hline Location & & & 0.21 \\
\hline Urban vs. rural & 1.23 & $0.89-1.68$ & \\
\hline Bed size & & & 0.35 \\
\hline Large vs. small & 1.18 & $0.94-1.48$ & \\
\hline Medium vs. small & 1.06 & $0.81-1.38$ & \\
\hline Region & & & 0.18 \\
\hline Midwest vs. northeast & 0.84 & $0.68-1.03$ & \\
\hline South vs. northeast & 0.84 & $0.70-1.03$ & \\
\hline West vs. northeast & 0.93 & $0.76-1.14$ & \\
\hline Charlson's comorbidity score & 1.17 & $1.13-1.21$ & $<0.0001$ \\
\hline Year & 0.96 & $0.92-0.99$ & 0.02 \\
\hline
\end{tabular}

\section{Sensitivity Analysis}

Excluding Those with Missing Race

We performed a sensitivity analysis by excluding those with missing values for race. Similar to the primary analysis, dialysis patients had two times higher odds of inhospital mortality (OR 1.99; 95\% CI: 1.6, 2.5) after adjusting for relevant covariates.

\section{Those with Sepsis/Infective Endocarditis ICD-9 CM} Codes

In the analysis restricted to those with ICD-9 codes for sepsis, the dialysis status was associated with 1.31 (95\% 
CI: 1.05, 1.65) higher odds of death and those with ICD-9 codes for endocarditis (even though there was a trend) were not significantly associated with higher odds of death (OR 1.35, 95\% CI: 0.99, 1.83).

\section{Discussion}

In this large representative database of hospitalizations in the United States, we observed an increase in the incidence of CIED-infection-related hospitalization over time among dialysis population. The unadjusted all-cause in-hospital mortality was $5.9 \%$ in non-dialysis patients and $13.7 \%$ in dialysis patients. In the multivariable model, two-fold increased odds of in-hospital death were observed in dialysis patients admitted with CIED-related infections. They also had a longer hospital stay; a higher proportion of them required transfer to an extended care facility at the time of discharge along with a higher cost of hospitalization compared to the non- dialysis group.

Rising rates of CIED-related infections have been described in the general population, associated with an increased risk of in-hospital mortality $[8,9]$. Among other factors, the presence of kidney disease, including being on dialysis, has been reported as an independent predictor of CIED infections as well as mortality $[9,13,17]$. We noted increasing rates of CIED-infection-related hospitalization over time in the dialysis population. Even though this increase might be related to the increasing number of procedures being done in the recent years, the increasing numbers of CIED-related infections impose a significant burden to patients and health care systems. Our findings confirm higher odds of in-hospital death following CIED infection-related hospitalization in the dialysis population. However, this was significantly lower than previously reported odds of in-hospital death attributed to renal failure. It is likely that the ICD-9-CM code for renal failure in previous studies may have incorporated both acute renal failure as well as non-dialysis-dependent CKD patients along with the dialysis population. On the other hand, this analysis is restricted to dialysis patients. Including data only from the year 2005 onwards (when the ICD-9-CM code for ESRD was introduced), we observed two-times increased odds for in-hospital mortality among dialysis patients hospitalized with CIED-related infections.

Kidney disease is a known risk multiplier for several conditions, more so in patients hospitalized with infections. Dialysis patients have been reported to have a higher incidence of infections including sepsis, pneumonia, orthopedic and endovascular infections [15, 18-21]. The increased risk is attributed to underlying uremia-related immune dysfunction, presence of temporary vascular access and transient bacteremia with repeated hemodialysis procedures $[15,22,23]$. These are in addition to other traditional factors such as older age, hypoalbuminemia, malnutrition and higher comorbidity burden that have been shown to be independently associated with infectious risk in this population $[24,25]$. They also have higher mortality rates following infection-related hospitalizations with $15 \%$ overall in-hospital mortality rate reported in hemodialysis patients. The risk of death varies depending on the source of infection, with 7\% all-cause, in-hospital mortality rate for vascular access infections, $17 \%$ for respiratory infections and up to $30 \%$ for cardiac infections [21]. Understanding the difference in the characteristics of the study population and definition used, our report of $13.7 \%$ mortality rate in dialysis patients following CIED infections may therefore seem modest in this high risk population.

The long-term implications of CIED infections in this population are however unclear. Systemic antimicrobial therapy, complete device and lead extraction followed by device re-implantation in an alternative location (if still indicated) are the recommended approaches for CIED infections [26]. However, dialysis patients have been shown to be less likely to be successfully re-implanted following biventricular device infections and in general, patients who were not successfully re-implanted, seemed to have poorer long-term outcomes [27]. While we were able to demonstrate an increased risk for in-hospital death with CIED-related infections, we could not examine the specific cause of death in this study. These increased deaths could be attributed to either underlying sepsis or increased risk for arrhythmic events in the dialysis population. An increased risk of cardiovascular events following acute infections has been previously described in the general population although the mechanism is not entirely clear [28]. One could speculate that such risk would also be amplified in the dialysis population. Further, the type of dialysis vascular access influences outcomes because patients with dialysis catheters have an increased risk for contracting infection and death when compared with patients who use arteriovenous fistulas for their dialysis access $[21,29,30]$. However, we were unable to identify the type of dialysis access from the NIS database; hence, we could not determine their effect on in-hospital mortality in the setting of CIED infections. Future studies could directly compare the incidence and outcomes of CIED infections to other types of infections (such as those related to vascular ac- 
cess, pneumonia etc.) in dialysis population to understand the severity and importance of various types of infections in the dialysis population.

Among survivors of CIED-related infections, we observed that the majority of patients would require extended care either at a long-term acute care facility, skilled nursing facility or other rehabilitation facilities. The cost associated with the additional level of care after discharge has not been previously reported and could not be evaluated in our study. However, it could be suggested that this disparity in discharge disposition would contribute to additional healthcare utilization cost in the dialysis population as well as increased rate of readmissions in those requiring a higher level of care after discharge.

Even though the cost of hospitalizations for CIED infections in the general population has been previously reported, the cost implication in specific risk groups has not been previously evaluated. Greenspon et al. had reported increased hospital charges for CIED infections in the general population, from about $\$ 75,000$ in 1993 to over $\$ 146,000$ by 2008 , representing a $47 \%$ increase per decade [9]. In our study, we have converted hospital charges to cost and observed $41 \%$ increased cost of hospitalization for dialysis patients when compared with that of the nondialysis patients hospitalized with CIED infections. These could be attributed to the increased length of stay among the dialysis population along with the additional laboratory and dialysis procedures performed during the hospitalization. In the adjusted analysis (including dialysis status as a variable), the higher costs among dialysis patients remains, highlighting the economic burden associated with CIED-related infections.

The main strength of this analysis includes the generalizability of the data and the availability of disposition details along with in-hospital costs. However, we recognize the limitations of this study being a retrospective analysis with inherent biases. We have used an administrative database that depended on ICD-9-CM codes to identify our study population. There may have been some degree of selection bias because coding may vary among different hospitals and individual coders. However, the coding variations are not expected to be substantially different when comparing dialysis and non-dialysis patients within the same hospital or individual coder. Moreover, these codes have been previously used in other studies evaluating CIED infections. Also, patients who were admitted with acute kidney failure warranting dialysis could have been included in the non-dialysis group and thus could have underestimated the risks associated with the dialysis population. Given the nature of the database used, we were not able to adjust for other predictors of mortality such as cardio-protective medication use and we did not have details relating to underlying cardiac function (EF, diastolic dysfunction, etc.). However, we have used the modified version of Charlson's comorbidity index to account for several comorbidities including the cardiovascular disease burden, which are often higher in dialysis patients.

In conclusion, in dialysis patients, hospitalization related to CIED-infections is increasing and is associated with an increased risk of in-hospital mortality, increased length of hospital stay and higher costs. Nevertheless, these rates are similar to mortality rates seen with other infections in this population and might not necessarily negate the potential benefits of CIED. Further studies are therefore needed to identify and modify specific risk factors for CIED-related infections within the dialysis population and ways to improve outcomes in those who are hospitalized with CIED infections.

\section{Disclosure Statements}

SDN was supported by a career development award from the National Center for Research Resources and the National Center for Advancing Translational Sciences, National Institutes of Health (Grant \# TR000440). JDS is supported by NIH/NIDDK (R01 DK085185 and DK094112) and investigator initiated-grant support from Genzyme Corporation, NIH/NIMH (P60MD00265), Health Services and Resources Administration (HRSA, 1R39OT 22056), the Centers for Disease Control and Prevention. The contents of this manuscript are solely the responsibility of the authors and do not necessarily represent the official views of the NIH. The authors have no relevant financial interest in the study. The results of this study were presented as an abstract at the Annual American Society of Nephrology meeting held on November 8, 2013 in Atlanta, GA, USA.

References

1 Cheung AK, Sarnak MJ, Yan G, Berkoben M, Heyka R, Kaufman A, Lewis J, Rocco M, Toto R, Windus D, Ornt D, Levey AS; HEMO Study Group: Cardiac diseases in maintenance hemodialysis patients: results of the HEMO study. Kidney Int 2004;65:23802389.

2 Stack AG, Bloembergen WE: Prevalence and clinical correlates of coronary artery disease among new dialysis patients in the United States: a cross-sectional study. J Am Soc Nephrol 2001;12:1516-1523.

3 Shamseddin MK, Parfrey PS: Sudden cardiac death in chronic kidney disease: epidemiology and prevention. Nat Rev Nephrol 2011;7: 145-154. 
4 Foley RN, Parfrey PS, Harnett JD, Kent GM, 14 Hickson LJ, Gooden JY, Le KY, Baddour LM, Martin CJ, Murray DC, Barre PE: Clinical and echocardiographic disease in patients starting end-stage renal disease therapy. Kidney Int 1995;47:186-192.

5 USRDS: 2013 Atlas of CKD and ESRD.

$\checkmark 6$ Herzog CA, Li S, Weinhandl ED, Strief JW, Collins AJ, Gilbertson DT: Survival of dialysis patients after cardiac arrest and the impact of implantable cardioverter defibrillators. Kidney Int 2005;68:818-825.

7 Garg N, Thomas G, Jackson G, Rickard J, Nally JV Jr, Tang WH, Navaneethan SD: Cardiac resynchronization therapy in CKD: a systematic review. Clin J Am Soc Nephrol 2013;8: 1293-1303.

8 Voigt A, Shalaby A, Saba S: Rising rates of cardiac rhythm management device infections in the United States: 1996 through 2003. J Am Coll Cardiol 2006;48:590-591.

9 Greenspon AJ, Patel JD, Lau E, Ochoa JA, Frisch DR, Ho RT, Pavri BB, Kurtz SM: 16year trends in the infection burden for pacemakers and implantable cardioverter-defibrillators in the United States 1993-2008. J Am Coll Cardiol 2011;58:1001-1006.

-10 Dasgupta A, Montalvo J, Medendorp S, Lloyd-Jones DM, Ghossein C, Goldberger J, Passman R: Increased complication rates of cardiac rhythm management devices in ESRD patients. Am J Kidney Dis 2007;49: 656-663.

11 Bloom H, Heeke B, Leon A, Mera F, Delurgio $\mathrm{D}$, Beshai J, Langberg J: Renal insufficiency and the risk of infection from pacemaker or defibrillator surgery. Pacing Clin Electrophysiol 2006;29:142-145.

12 Charytan DM, Patrick AR, Liu J, Setoguchi S, Herzog CA, Brookhart MA, Winkelmayer WC: Trends in the use and outcomes of implantable cardioverter-defibrillators in patients undergoing dialysis in the United States. Am J Kidney Dis 2011;58:409-417.

13 Tompkins C, McLean R, Cheng A, Brinker JA, Marine JE, Nazarian S, Spragg DD, Sinha $\mathrm{S}$, Halperin H, Tomaselli GF, Berger RD, Calkins H, Henrikson CA: End-stage renal disease predicts complications in pacemaker and ICD implants. J Cardiovasc Electrophysiol 2011;22:1099-1104. Friedman PA, Hayes DL, Wilson WR, Steckelberg JM, Sohail MR: Clinical presentation and outcomes of cardiovascular implantable electronic device infections in hemodialysis patients. Am J Kidney Dis. 2014;64:104-110.

15 Manian FA: Vascular and cardiac infections in end-stage renal disease. Am J Med Sci 2003; 325:243-250.

16 Deyo RA, Cherkin DC, Ciol MA: Adapting a clinical comorbidity index for use with ICD9-CM administrative databases. J Clin Epidemiol 1992;45:613-619.

17 Romeyer-Bouchard C, Da Costa A, Dauphinot V, Messier M, Bisch L, Samuel B, Lafond P, Ricci P, Isaaz K: Prevalence and risk factors related to infections of cardiac resynchronization therapy devices. Eur Heart J 2010;31:203210.

18 Abbott KC, Agodoa LY: Hospitalizations for bacterial endocarditis after initiation of chronic dialysis in the United States. Nephron 2002;91:203-209.

19 Sakalkale DP, Hozack WJ, Rothman RH: Total hip arthroplasty in patients on long-term renal dialysis. J Arthroplasty 1999;14:571575.

20 Sarnak MJ, Jaber BL: Pulmonary infectious mortality among patients with end-stage renal disease. Chest 2001;120:1883-1887.

21 Allon M, Depner TA, Radeva M, Bailey J, Beddhu S, Butterly D, Coyne DW, Gassman JJ Kaufman AM, Kaysen GA, Lewis JA, Schwab SJ; HEMO Study Group: Impact of dialysis dose and membrane on infection-related hospitalization and death: results of the HEMO study. J Am Soc Nephrol 2003;14:1863-1870.

22 Jaber BL: Bacterial infections in hemodialysis patients: pathogenesis and prevention. Kidney Int 2005;67:2508-2519.

23 Keane WF, Shapiro FL, Raij L: Incidence and type of infections occurring in 445 chronic hemodialysis patients. Trans Am Soc Artif Intern Organs 1977;23:41-47.
24 Dalrymple LS, Go AS: Epidemiology of acute infections among patients with chronic kidney disease. Clin J Am Soc Nephrol 2008;3: 1487-1493.

25 Dalrymple LS, Johansen KL, Chertow GM Cheng SC, Grimes B, Gold EB, Kaysen GA: Infection-related hospitalizations in older patients with ESRD. Am J Kidney Dis 2010;56: 522-530.

26 Baddour LM, Epstein AE, Erickson CC, Knight BP, Levison ME, Lockhart PB, Masoudi FA, Okum EJ, Wilson WR, Beerman LB, Bolger AF, Estes NA 3rd, Gewitz M, Newburger JW, Schron EB, Taubert KA; American Heart Association Rheumatic Fever, Endocarditis, and Kawasaki Disease Committee, Council on Cardiovascular Disease in Young, Council on Cardiovascular Surgery and Anesthesia, Council on Cardiovascular Nursing, Council on Clinical Cardiology, Interdisciplinary Council on Quality of Care, American Heart Association: Update on cardiovascular implantable electronic device infections and their management: a scientific statement from the American heart association. Circulation 2010;121:458-477.

27 Rickard J, Tarakji K, Cheng A, Spragg D, Cantillon DJ, Martin DO, Baranowski B, Gordon SM, Tang WH, Kanj M, Wazni O, Wilkoff BL: Survival of patients with biventricular devices after device infection, extraction, and reimplantation. JACC Heart Fail 2013;1:508-513.

28 Smeeth L, Thomas SL, Hall AJ, Hubbard R, Farrington P, Vallance P: Risk of myocardial infarction and stroke after acute infection or vaccination. N Engl J Med 2004;351:2611-2618.

29 Stevenson KB, Hannah EL, Lowder CA, Adcox MJ, Davidson RL, Mallea MC, Narasimhan N, Wagnild JP: Epidemiology of hemodialysis vascular access infections from longitudinal infection surveillance data: predicting the impact of NKF-DOQI clinical practice guidelines for vascular access. Am J Kidney Dis 2002;39:549-555.

30 Ravani P, Palmer SC, Oliver MJ, Quinn RR, MacRae JM, Tai DJ, Pannu NI, Thomas C, Hemmelgarn BR, Craig JC, Manns B, Tonelli M, Strippoli GF, James MT: Associations between hemodialysis access type and clinical outcomes: a systematic review. J Am Soc Nephrol 2013;24:465-473. 